\title{
Angiotensin-(1-7) Rescues Chronic Intermittent Hypoxia-Aggravated Transforming Growth Factor- $\beta$-Mediated Airway Remodeling in Murine and Cellular Models of Asthma
}

\author{
Jian Ping Zhou, ${ }^{1}$ Ying Ni Lin, ${ }^{1}$ Ning Li, Xian Wen Sun, Yong Jie Ding, Ya Ru Yan, \\ Liu Zhang, and Qing Yun Li \\ Department of Respiratory and Critical Care Medicine, Ruijin Hospital and Institute of Respiratory Diseases, Shanghai Jiao Tong \\ University School of Medicine, Shanghai, China
}

Received June 5, 2020; accepted August 25, 2020

\begin{abstract}
Renin-angiotensin system (RAS) is involved in TGF- $\beta$-mediated epithelial-to-mesenchymal transition (EMT) and is responsible for airway remodeling in refractory asthma. Obstructive sleep apnea (OSA), which affects RAS activity, is a risk factor for refractory asthma. We aimed to investigate how chronic intermittent hypoxia $(\mathrm{IH})$, the main pathophysiology of OSA, exacerbates asthma and whether Ang-(1-7) protects against chronic IH-induced airway remodeling in asthma. We exposed ovalbumin (OVA)-challenged asthma mice to chronic $\mathrm{IH}$ and observed that chronic $\mathrm{IH}$ aggravated airway inflammation and collagen deposit in OVA-challenged mice. Compared with the OVA group, the OVA + chronic IH group had a lower expression level of epithelial marker E-cadherin and higher expression levels of mesenchymal markers $\alpha$-smooth muscle actin and collagen IV in airway epithelia, accompanied with activation of TGF- $\beta /$ Smad pathway. These changes were reversed by the administration of Ang-(1-7). Consistently, Ang-(1-7) mitigated chronic $\mathrm{IH}$-induced activation of
\end{abstract}

TGF- $\beta$-mediated EMT in lipopolysaccharide-treated bronchial epithelial cells in a dose-dependent manner, which was blocked by Ang-(1-7)-specific Mas receptor antagonist A779. Taken together, Ang-(1-7) rescued chronic $\mathrm{IH}$-aggravated TGF- $\beta$-mediated EMT to suppress airway remodeling, implying that RAS activity is involved in the mechanisms of OSArelated airway dysfunction in asthma.

\section{SIGNIFICANCE STATEMENT}

OSA is a risk factor for refractory asthma. In this study, we aimed to explore the mechanisms of how OSA exacerbates refractory asthma. We found that chronic IH induces TGF- $\beta$-mediated EMT and aggravates airway collagen deposit. We also found that Ang-(1-7) erased the aggravation of TGF- $\beta$-mediated EMT and epithelial fibrosis upon chronic $\mathrm{IH}$ exposure. These findings provided new insights that the ACE2/Ang-(1-7)/Mas axis might be considered as a potential therapeutic target for patients with asthma and OSA.

\section{Introduction}

Asthma is a common chronic respiratory disease affecting $1 \%-18 \%$ of the population in different countries (GINA, 2020). A study in the Netherlands shows that difficult-tocontrol asthma accounts for about $17.4 \%$ of patients with asthma (Hekking et al., 2015), causing increased morbidity and economic burden. Airway remodeling is associated with irreversible decline of lung function, decreased bronchodilator reversibility, and increased airway hyper-responsiveness in refractory asthma (Trevor and Deshane, 2014). TGF- $\beta$-induced

We would like to acknowledge funding grants from the National Natural Science Foundation of China [81700085, 81700084], National Key R\&D Program of China [2018YFC1311900], and Shanghai Key Discipline for Respiratory Disease [2017ZZ02014] and support from Innovative research team of high-level local universities in Shanghai.

All authors disclose no conflicts of interest.

${ }^{1}$ J.P.Z. and Y.N.L. contributed equally to this article as co-first authors

https://doi.org/10.1124/jpet.120.000150. epithelial-to-mesenchymal transition (EMT) mediates the differentiation of airway epithelia into myofibroblasts to promote subepithelial fibrosis and thereby plays an essential role in asthmatic airway remodeling (Hackett, 2012). The reninangiotensin system (RAS) is involved in EMT induction. The activity of RAS depends on the balance between the ACE/AngII/AT1 and ACE2/Ang-(1-7)/Mas axes (Ferreira and Santos, 2005). During severe asthma attacks, the plasma levels of the two major RAS components, renin and angiotensin II (AngII), are increased (Millar et al., 1994). Exogenous angiotensin-(1-7) [Ang-(1-7)], which counterbalances AngII actions, represses fibroblast-myofibroblast transition and may inhibit airway remodeling in asthma via TGF- $\beta /$ Smad pathway (Zhou et al., 2016). Moreover, exogenous Ang-(1-7) treatment inhibits EMT and pulmonary fibrosis (Shao et al., 2019). Therefore, the unbalanced expression of AngII/Ang(1-7) may be involved in EMT induction to promote airway remodeling.

ABBREVIATIONS: ACE, Angiotensin-converting enzyme; Ang-(1-7), angiotensin-(1-7); Angll, angiotensin II; BALF, bronchoalveolar lavage fluid; EMT, epithelial-to-mesenchymal transition; IH, intermittent hypoxia; IL, interleukin; LPS, lipopolysaccharide; Mas, G-coupled protein receptor of angiotensin-(1-7); OSA, obstructive sleep apnea; OVA, ovalbumin; RAS, renin-angiotensin system; $\alpha$-SMA, $\alpha$-smooth muscle actin; Smad, drosophila mothers against decapentaplegic protein; TGF- $\beta$, transforming growth factor- $\beta$. 
Obstructive sleep apnea (OSA), which is characterized by recurrent collapse of the upper airway during sleep, shows a high prevalence in patients with refractory asthma (Yigla et al., 2003). OSA has been reported as a risk factor for frequent exacerbations in refractory asthma, with an adjusted odds ratio of 3.4 (ten Brinke et al., 2005). The underlying mechanisms of OSA-related aggravation of asthma are poorly understood. Previous studies showed that chronic intermittent hypoxia (IH), the main physiologic process of OSA, causes bronchial hyperreactivity, enhances airway and systemic inflammation, and thus increases the risk of refractory asthma. Notably, chronic IH induces sympathetic nerve activation, increases serum level of AngII, and activates RAS, which may result in vascular injury and remodeling. Exogenous Ang-(1-7) could attenuate chronic IH-induced lung injury via inhibition of inflammation and oxidative stress (Lu et al., 2016) and could relieve chronic $\mathrm{IH}$-induced renal injury by reducing fibrosis ( $\mathrm{Lu}$ et al., 2017). However, whether chronic IH aggravates airway remodeling via affecting RAS activity and whether Ang-(1-7) protects against chronic IH-induced airway remodeling in asthma remains unknown.

Therefore, we hypothesized that chronic IH aggravates TGF- $\beta$-mediated EMT and airway remodeling in asthma via RAS activation. To test the hypothesis, we analyzed expression levels of EMT-related proteins and the activity of TGF- $\beta$ /Smad pathway in a chronic IH-exposed ovalbumin (OVA)-induced asthma mouse model and lipopolysaccharide (LPS)-induced pulmonary epithelial cells exposed to IH condition. The mice or cells were treated with Ang-(1-7) to illustrate whether RAS activity is involved in chronic IH-induced airway remodeling similar to that in asthma. Our findings provide a new insight in the mechanisms of how chronic IH aggravates asthma.

\section{Materials and Methods}

Animal Models. Eight-week-old male C57BL/6 mice weighing 20-22 g were purchased from SLAC Laboratory Animal Co. Ltd. (Shanghai, China). They were housed under specific pathogen-free conditions and were kept on an ovalbumin-free diet. The animal study was approved by the Committee for Research and Ethics of Ruijin Hospital, affiliated with Shanghai Jiao Tong University School of Medicine, in accordance with the Guide for the Care and Use of Laboratory Animals (eighth edition, 2011, published by The National Academies Press, 2101 Constitution Ave. NW, Washington, DC 20055). All animal work was done in Ruijin Hospital, Shanghai Jiao Tong University School of Medicine. All surgeries were performed under sodium pentobarbital anesthesia, and all efforts were made to minimize suffering.

The mice were divided into four groups: control, OVA, OVA + chronic $\mathrm{IH}$, and OVA + chronic IH + Ang-(1-7). They were sensitized intraperitoneally with $0.2 \mathrm{mg}$ OVA (grade V; Sigma, St Louis, MO) complexed with $10 \mathrm{mg}$ of alum in a total volume of $1 \mathrm{ml}$ saline on day 0 and day 7 . Mice from the control group were injected with alum in $1 \mathrm{ml}$ saline. The control and OVA groups were exposed to intermittent air, and the OVA + chronic IH and OVA + chronic IH + Ang-(1-7) groups were exposed to chronic $\mathrm{IH}\left(14 \% \mathrm{O}_{2}\right.$ for 5 seconds followed by $21 \% \mathrm{O}_{2}, 30$ cycles per hour, 8 hours/day) from day 14 to day 42. In the OVA + chronic IH + Ang-(1-7) group, mice received an intraperitoneal injection of Ang-(1-7) (Sigma) at a concentration of $150 \mu \mathrm{g} / \mathrm{kg}$ on days $14,21,28,35$, and 42 .

Bronchoalveolar Lavage Fluid Collection. Twenty-four hours after the last chronic IH exposure, mice were sacrificed by an overdose administration of pentobarbital ( $100 \mathrm{mg} / \mathrm{kg}$ intraperitoneal), and the lungs were isolated by blunt dissection. Three successive volumes of
$1 \mathrm{ml}$ phosphate-buffered saline were instilled via the endotracheal tube and aspirated gently, and bronchoalveolar lavage fluid (BALF) was pooled. Each BALF sample was centrifuged at $1000 \mathrm{~g}$ for 10 minutes at $4^{\circ} \mathrm{C}$, and the supernatants were stored at $-80^{\circ} \mathrm{C}$ until use. Cell pellets were diluted with $0.5 \mathrm{ml}$ of phosphate-buffered saline. Total cell counts were determined using a hemocytometry by adding $100 \mu \mathrm{l}$ of the cell suspension to $100 \mu \mathrm{l}$ of $0.4 \%$ trypan blue. Differential cell counts were performed on cytocentrifuge preparations (Cytospin 2; Shandon Instruments, Runcorn, UK) stained with Wright-Giemsa, and 200 cells were counted under $\times 400$ magnification by three different investigators in a blinded manner. Cells were identified by standard morphology and classified as macrophages, lymphocytes, neutrophils, and eosinophils. The levels of inflammatory cytokines interleukin (IL)- 6 and IL- $1 \beta$ in BALF were determined using an ELISA kit (Nanjing Jiancheng Bioengineering Institute) according to standard protocols.

Lung Histologic Analysis. The left lung lobe was fixed in $10 \%$ neutral-buffered formalin solution and embedded into paraffin. Lung sections $(5 \mu \mathrm{m})$ underwent $\mathrm{H} \& \mathrm{E}$ staining and Masson staining as previously described (Wang et al., 2016; Du et al., 2017).

Immunofluorescence Staining. Samples were deparaffinized with xylol, sliced into $4-\mu \mathrm{m}$ sections, and rehydrated using a graded ethanol series. A heat-induced epitope protocol was used for antigen retrieval $\left(95^{\circ} \mathrm{C}\right.$ for 40 minutes). Samples were incubated in methanol containing $0.3 \%$ hydrogen peroxide to block endogenous peroxidases. Samples were blocked with protein serum using a Vectastain Elite $\mathrm{ABC}$ kit (Vector Laboratories, Inc., Burlingame, CA) and then incubated overnight at $4^{\circ} \mathrm{C}$ with anti- $\alpha$-smooth muscle actins ( $\alpha$-SMA; \#19245) or anti-E-cadherin (\#14472) antibody (Cell Signaling Technology, Danvers, MA) at 1:1000 dilutions. After washing three times in Tris-buffered saline/Tween $20(150 \mathrm{mM} \mathrm{NaCl}, 10 \mathrm{mM}$ Tris-HCl, $\mathrm{pH}$ 7.6), sections were incubated with a secondary antibody for 20 minutes at room temperature. Peroxidase-conjugated biotin-streptavidin complex (Dako, Glostrup, Denmark) was then applied to sections for 20 minutes. Sections were visualized with $3,3^{\prime}$-diaminobenzidine and counterstained with hematoxylin. For the negative control, nonimmune serum was used instead of primary antibody.

Cell Culture. Human bronchial epithelial cells, BEAS-2B, were obtained from the American Type Culture Collection (Rockville, MD) and were cultured in Dulbecco's modified Eagle's medium containing $10 \%$ fetal calf serum at $37^{\circ} \mathrm{C}$ with $5 \% \mathrm{CO}_{2}$ and $95 \%$ air. The cells were then treated with LPS $(10 \mu \mathrm{g} / \mathrm{ml})$. Either Ang-(1-7) $(100 \mathrm{nM})$ or A779 $(100 \mathrm{nM})$ (Sigma) was added 30 minutes before LPS $(10 \mu \mathrm{g} / \mathrm{ml})$ treatment of 48 hours. For IH exposure, cells were exposed to 5 seconds of $14 \%$ to $15 \% \mathrm{O}_{2}$ during every 60 -second cycle for 24 hours.

Protein Extraction and Western Blotting. Proteins were extracted from subconfluent cell cultures and subjected to Western blot analyses. After blocking with 5\% nonfat milk in phosphate buffered solution Tween for 1 hour at room temperature, the membranes were blotted with primary antibody, followed by blocking and incubation with a peroxidase-conjugated secondary antibody. Bound antibodies were visualized using enhanced chemiluminescence (Bio-Rad, Hercules, CA). The antibodies were purchased from Cell Signaling Technology, the primary antibodies against $\alpha$-SMA (\#19245), Snail (\#3879), E-cadherin (\#14472), Smad2 (\#5339), phosphor-Smad2 (\#18338), TGF- $\beta$ (\#3711), and $\beta$-actin (\#4970). Signals were detected using a FluorChem E system (Alpha Innotech Corp, Santa Clara, CA). All Western blotting experiments were performed in triplicate.

Statistical Analysis. The results are expressed as means \pm S.D. One-way ANOVA was used for comparison among multiple groups, and Tukey's post hoc test was performed for comparison between two groups. $P<0.05$ denotes statistical significance.

\section{Results}

Ang-(1-7) Protected against Chronic IH-Aggravated Lung Injury in OVA-Challenged Mice. To investigate the effect of chronic IH on asthma aggravation, we exposed the OVA-induced asthma mouse model to the chronic IH condition 
for 28 days. Compared with the OVA group, the OVA + chronic IH group presented with an increase in inflammatory cell infiltration (Fig. 1A) and collagen deposition (Fig. 1B), which were partially mitigated by administration of Ang-(1-7) (150 $\mu \mathrm{g} / \mathrm{kg}$ ) (Fig. 1, A and B). Moreover, the more severe lung injury in the OVA + chronic IH group was accompanied with marked increased accounts of lymphocytes (OVA: $7.4 \pm 0.8 \times 10^{9} / \mathrm{l}$ vs. OVA + chronic IH: $9.5 \pm 1.4 \times 10^{9} / \mathrm{l}, P=0.002$ ), macrophages (OVA: $13.5 \pm 1.7 \times 10^{9} /$ vs. OVA + chronic IH: $24.3 \times \pm 2.8 \times$ $10^{9} /, P<0.001$ ), and eosinophils (OVA: $5.6 \pm 0.4 \times 10^{9} / \mathrm{l}$ vs. OVA + chronic IH: $\left.7.3 \pm 0.7 \times 10^{9} /, P<0.001\right)$ and higher levels of IL-6 (OVA: $168.5 \pm 25.5 \mathrm{pg} / \mathrm{ml}$ vs. OVA + chronic IH: $196.5 \pm 32.1 \mathrm{pg} / \mathrm{ml}, P=0.024$ ) and IL- $\beta$ (OVA: $102.1 \pm 13.0$ $\mathrm{pg} / \mathrm{ml}$ vs. OVA + chronic IH: $117.1 \pm 14.3 \mathrm{pg} / \mathrm{ml}, P=0.028)$ in BALF (Fig. 1, C and D). All these changes were reversed by administration of Ang-(1-7) (Fig. 1, C and D). The results indicated that chronic IH amplifies airway inflammation and remodeling in OVA-challenged mice, which is attenuated by Ang-(1-7).

Ang-(1-7) Suppressed Chronic IH-Induced Activation of TGF- $\beta$-Mediated EMT in OVA-Challenged Mice. Since EMT may contribute to airway remodeling in asthma, we then verified whether chronic IH facilitates EMT in OVA-challenged mice. Immunofluorescence staining showed the OVA group presented with a loss in expression of epithelial marker E-cadherin and a gain in expression of mesenchymal markers $\alpha$-SMA and collagen IV in airway epithelia (Fig. 2, A and B). The results were confirmed by Western blotting (Fig. 2C). These changes became more pronounced in the OVA + chronic IH group (Fig. 2, A-C). Moreover, administration of Ang-(1-7) significantly upregulated the expression of E-cadherin and downregulated the expression of $\alpha$-SMA and collagen IV in the OVA + chronic IH group (Fig. 2, A-C).

TGF- $\beta$ promotes Smad phosphorylation, induces the expression of transcription factor Snail, and subsequently represses E-cadherin expression, resulting in EMT induction [15]. OVA challenge induced the expression of TGF- $\beta$ and phosphorylation of Smad (Fig. 2D). Compared with the OVA group, the levels of TGF- $\beta$, phosphorylated Smad, and Snail were further increased in the OVA + chronic IH group (Fig. 2D), which was reversed by administration of Ang-(1-7) (Fig. 2D). Taken together, Ang-(1-7) suppresses chronic IH-promoted TGF- $\beta$-mediated EMT in OVA-challenged mice.

Ang-(1-7) Mitigated IH-Induced Activation of TGF- $\beta$ Mediated EMT in LPS-Treated Bronchial Epithelial Cells. To confirm the effects of IH on EMT induction in vitro, we exposed LPS $(10 \mu \mathrm{g} / \mathrm{ml})$-treated bronchial epithelial cells (BEAS-2B) to the IH condition. LPS caused a decrease in E-cadherin expression, an increase in $\alpha$-SMA expression, and an activation of TGF- $\beta$ /Smad pathway in BEAS-2B cells (Fig. 3, A-C). Compared with the LPS group, the LPS + IH group showed a much lower E-cadherin level and a higher $\alpha$-SMA level (Fig. 3, A-C), as well as increased levels of TGF- $\beta$, phosphorylated Smad, and Snail, which was attenuated by pretreatment of Ang-(1-7) in a dose-dependent manner (Fig. 3D). Since Ang-(1-7) interacts with the downstream G protein-coupled receptor Mas to oppose the effects of angiotensin II, we pretreated the cells with the Ang-(1-7)-specific Mas receptor antagonist A779. We found that A779 (100 nM) blocked the suppressive effects of Ang-(1-7) on EMT upon IH exposure, presented as a decrease in E-cadherin expression and an increase in $\alpha$-SMA expression in LPS + IH + Ang-(1-7) + A779 group compared with LPS + IH + Ang-(1-7) group (Fig. 4, A-C). Moreover, A779 attenuated the inhibitory effect of Ang-(1-7) on activation of the TGF- $\beta /$ Smad pathway induced by IH exposure (Fig. 4D). These results confirmed that Ang-(1-7) rescues IH-induced TGF- $\beta$-mediated EMT in LPS-treated bronchial epithelial cells.

\section{Discussion}

In recent years, the prevalence of asthma has been increasing worldwide. OSA has been reported to be a risk factor for refractory asthma. However, how OSA aggravates asthma remains unknown. Chronic IH contributes to the cardiovascular and metabolic consequences of OSA (Sforza and Roche, 2016). In this study, we provided evidence that chronic IH accentuated airway inflammation and collagen deposition in OVA-challenged mice and promoted TGF- $\beta$-mediated EMT, which was reversed by the administration of Ang-(1-7). These results suggest that chronic IH induces TGF- $\beta$-mediated EMT, which may involve the ACE2/Ang-(1-7)/Mas axis, and thereby contributes to asthma aggravation.

Asthma is characterized by persistent airway inflammation and airway wall remodeling. EMT could cause airway remodeling. Previous studies have shown airway subepithelial fibrosis and downregulated expression levels of epithelial marker E-cadherin and upregulated expression levels of mesenchymal markers $\alpha$-SMA and collagen IV in airway epithelia of OVA-challenged mice and in patients with asthma (Liu et al., 2017; Tian et al., 2017). TGF- $\beta$ is a profibrotic cytokine that plays an important role in EMT induction and airway remodeling in asthma (Qu et al., 2012; Tian et al., 2017). After TGF- $\beta$ binds to its receptor, Smad is phosphorylated and activated, and sequentially the expression of transcription factor Snail is upregulated. Snail binds to E-boxes in the promoter of the E-cadherin gene and downregulates the expression of E-cadherin, which is considered to be characteristic of EMT (Yang et al., 2013). Consistently, we observed that, compared with the control group, the OVA group presented with increased airway collagen deposit, lower levels of E-cadherin, higher levels of $\alpha$-SMA and collagen IV, and activation of TGF- $\beta /$ Smad pathway. The results were confirmed in LPS-treated bronchial epithelial cells.

To explore the underlying mechanisms of how OSA exacerbates asthma, we exposed the OVA-challenged mice to chronic IH at a rate of 30 cycles per hour, which mimics the severity of OSA observed in patients with asthma (Julien et al., 2009; Broytman et al., 2015). The nadir $\mathrm{O}_{2}$ concentration in our protocol was relatively higher than that reported in previous studies. This was because OVAchallenged mice in the study were more sensitive to hypoxia, and more than half of the OVA-challenged mice died within the first 30 minutes after IH exposure began on the first round. We observed that chronic IH augmented airway collagen density in OVA-challenged mice, which was consistent with a previous study reporting that chronic IH induces collagen deposition and matrix degradation and causes airflow limitation in OVA-challenged BrownNorway rats (Meng et al., 2015). Then, we found that chronic 
A Control OVA $\mathrm{OVA}+\mathrm{ClH}$ $\mathrm{OVA}+\mathrm{ClH}$
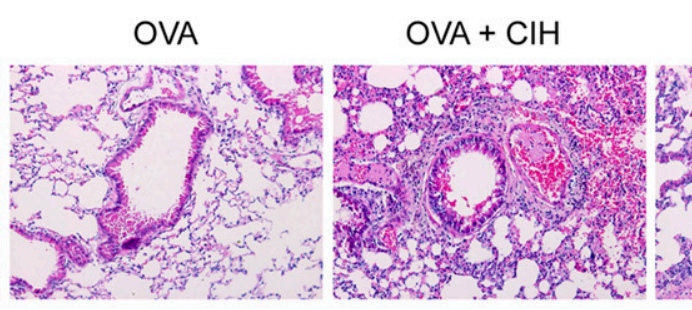

+Ang-(1-7)

B Control OVA

$\mathrm{OVA}+\mathrm{ClH}$

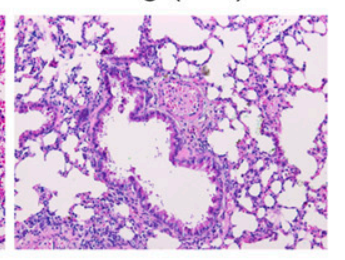

B
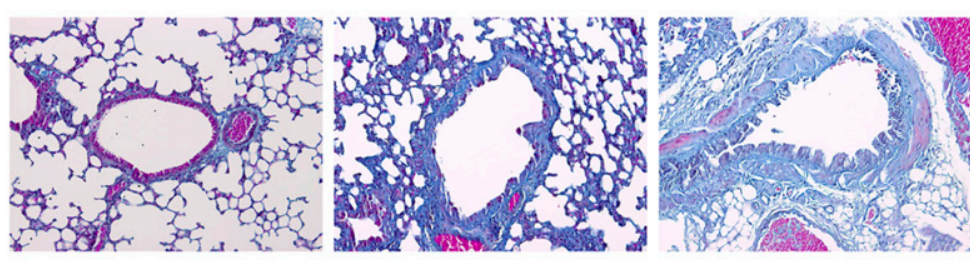

$$
\mathrm{OVA}+\mathrm{ClH}
$$

+ Ang-(1-7)

C
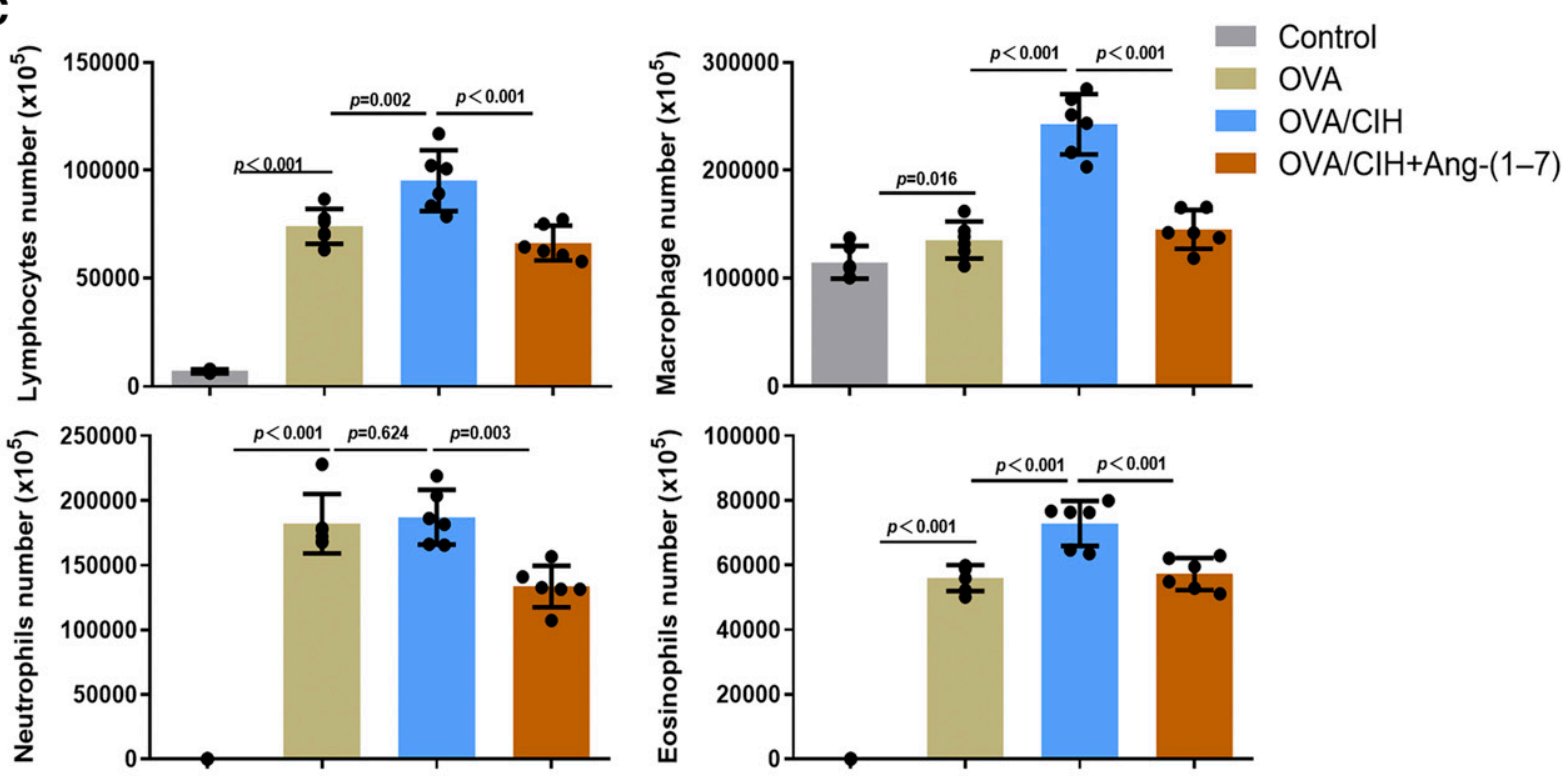

\section{D}
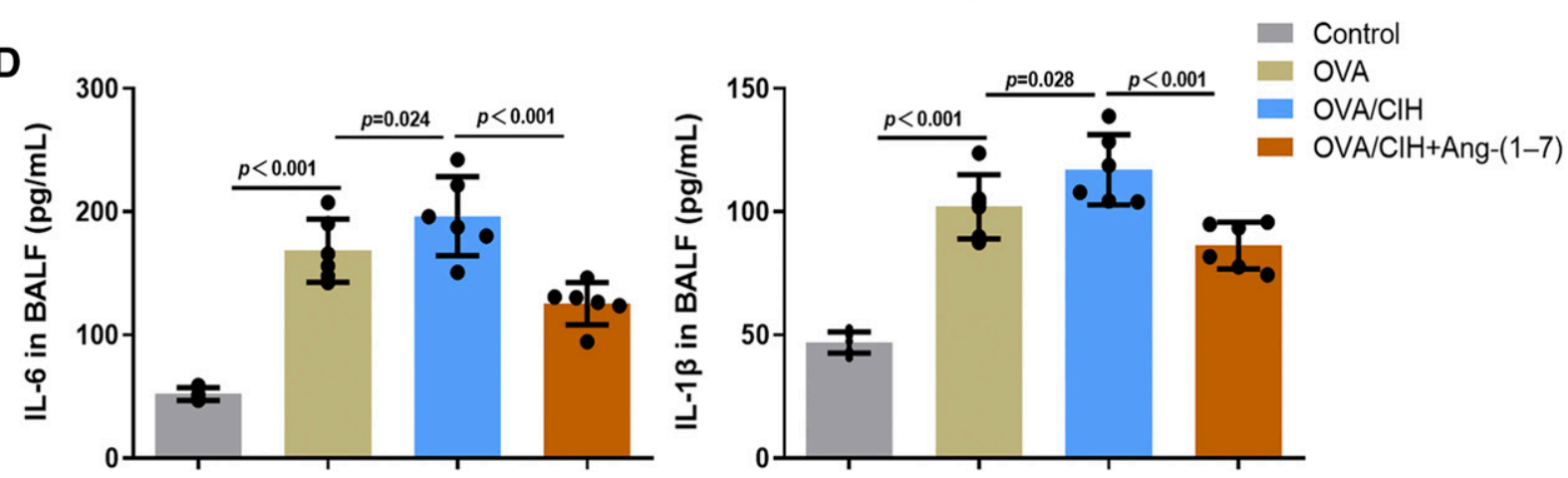

Fig. 1. Ang-(1-7) attenuated chronic IH-induced airway inflammation and collagen deposit in OVA-sensitized mice. (A) Representative images of H\&E staining of lung tissue of the mice showed an increase in inflammatory cell infiltration in the OVA + chronic IH group compared with OVA group, which was attenuated by administration of Ang-(1-7) $(150 \mu \mathrm{g} / \mathrm{kg})$. Scale bar, $100 \mu \mathrm{m}$. (B) Representative Masson staining of lung tissues of the mice showed an increase in collagen deposit in the OVA + chronic IH group compared with OVA group, which was attenuated by administration of Ang-(1-7) (150 $\mu \mathrm{g} / \mathrm{kg})$. Scale bar, $100 \mu \mathrm{m}$. (C) Differential cell counts in BALF of the mice. (D) The levels of inflammatory factors IL-6 and IL-1 $\beta$ in BALF of the mice as measured using ELISA. Data are expressed as means \pm S.D. $(n=6) . * P:<0.05 ; * * P<0.01 ; * * * P<0.001$ by one-way ANOVA followed by Tukey's multiple comparison test. 
A

A Control
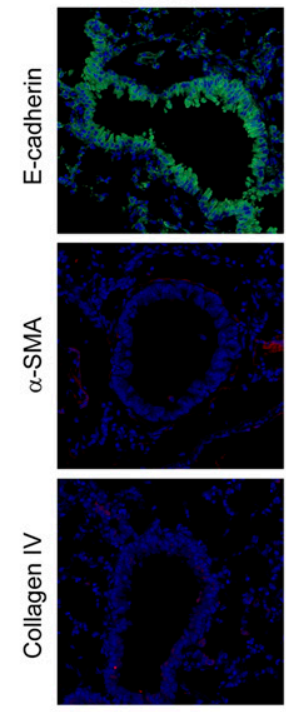

B
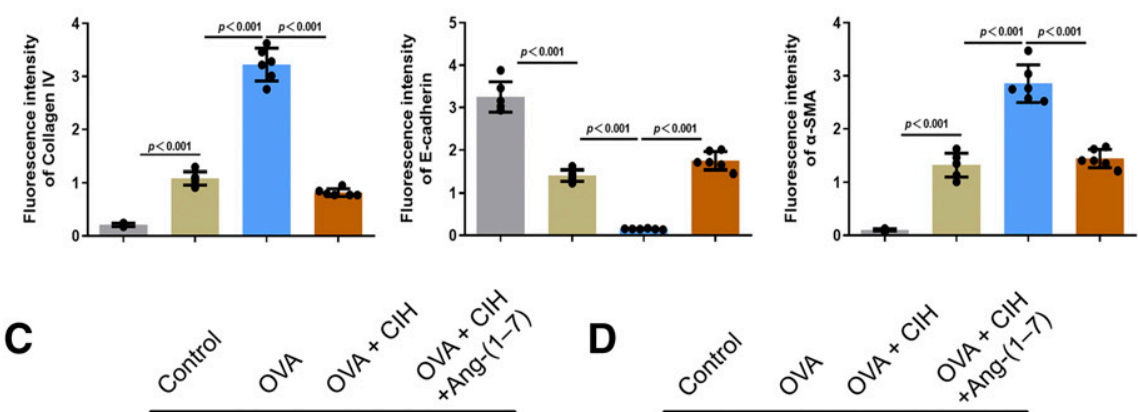

E-cadherin

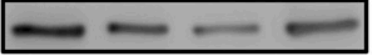

$\alpha-\mathrm{SMA}$

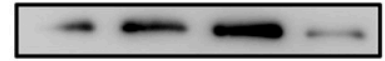

Collagen IV

$\beta$-actin
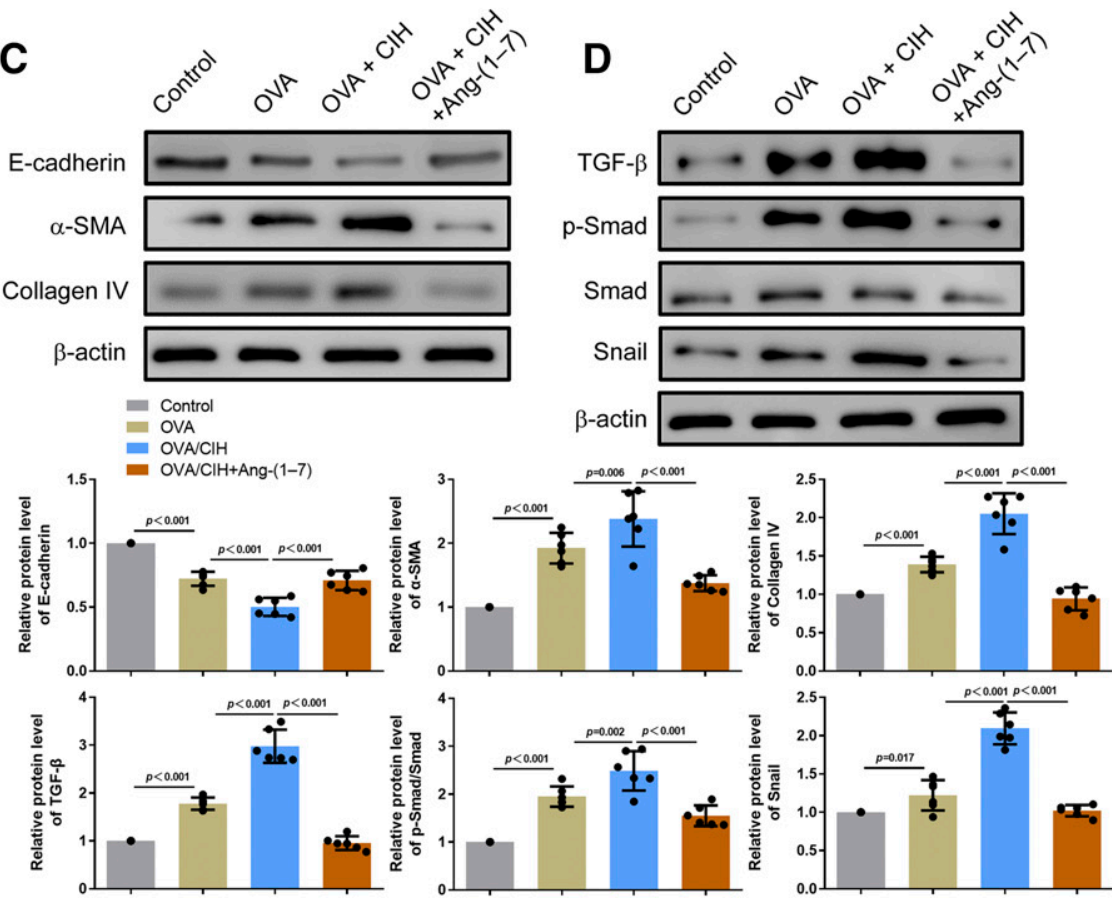

Fig. 2. Ang-(1-7) suppressed chronic IH-aggravated TGF- $\beta$-mediated EMT in OVA-sensitized mice. (A) Chronic IH decreased the level of epithelial marker E-cadherin and increased levels of mesenchymal markers $\alpha$-SMA and collagen IV in airway epithelia of OVA-sensitized mice, which was reversed by administration of Ang-(1-7) (150 $\mu \mathrm{g} / \mathrm{kg})$. Representative images of immunofluorescence staining of lung tissues of the mice with antibodies against E-cadherin, $\alpha$-SMA, and collagen IV were shown. Scale bar, $100 \mu \mathrm{m}$. (B) The average fluorescence intensity of E-cadherin, $\alpha$-SMA, and collagen IV was shown. Data are expressed as means \pm S.D. $(n=6) . * P:<0.05$; **P $<0.01 ; * * * P<0.001$ by oneway ANOVA followed by Tukey's multiple comparison test. (C) Representative Western blots with antibodies against E-cadherin, $\alpha$-SMA, and collagen IV in the indicated groups. $\beta$-Actin was used as the internal control. (D) Chronic IH increased the levels of TGF- $\beta$, p-Smad, and Snail in lung tissues of OVA-sensitized mice, which was reversed by administration of Ang-(1-7) (150 $\mu \mathrm{g} / \mathrm{kg})$. Representative Western blots with antibodies against TGF$\beta$, p-Smad, Smad, and Snail were shown. $\beta$-Actin was used as the internal control.
IH reduced levels of epithelial marker E-cadherin and increased levels of mesenchymal markers $\alpha$-SMA and collagen IV in airway epithelia of OVA-challenged mice and also amplified the effect of OVA on activation of the TGF- $\beta / \mathrm{Smad}$ pathway, indicating that chronic IH may promote EMT and airway remodeling via activation of the TGF- $\beta /$ Smad pathway in asthma.

RAS activity is involved in airway remodeling in asthma. We previously found that RAS plays a key role in epithelial fibrosis via the TGF- $\beta$ /Smad pathway (Zhou et al., 2016). The 
A

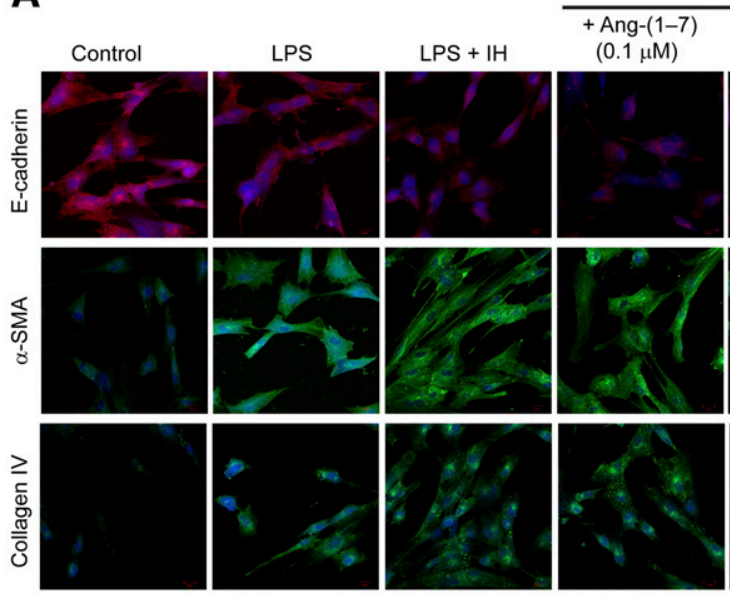

B
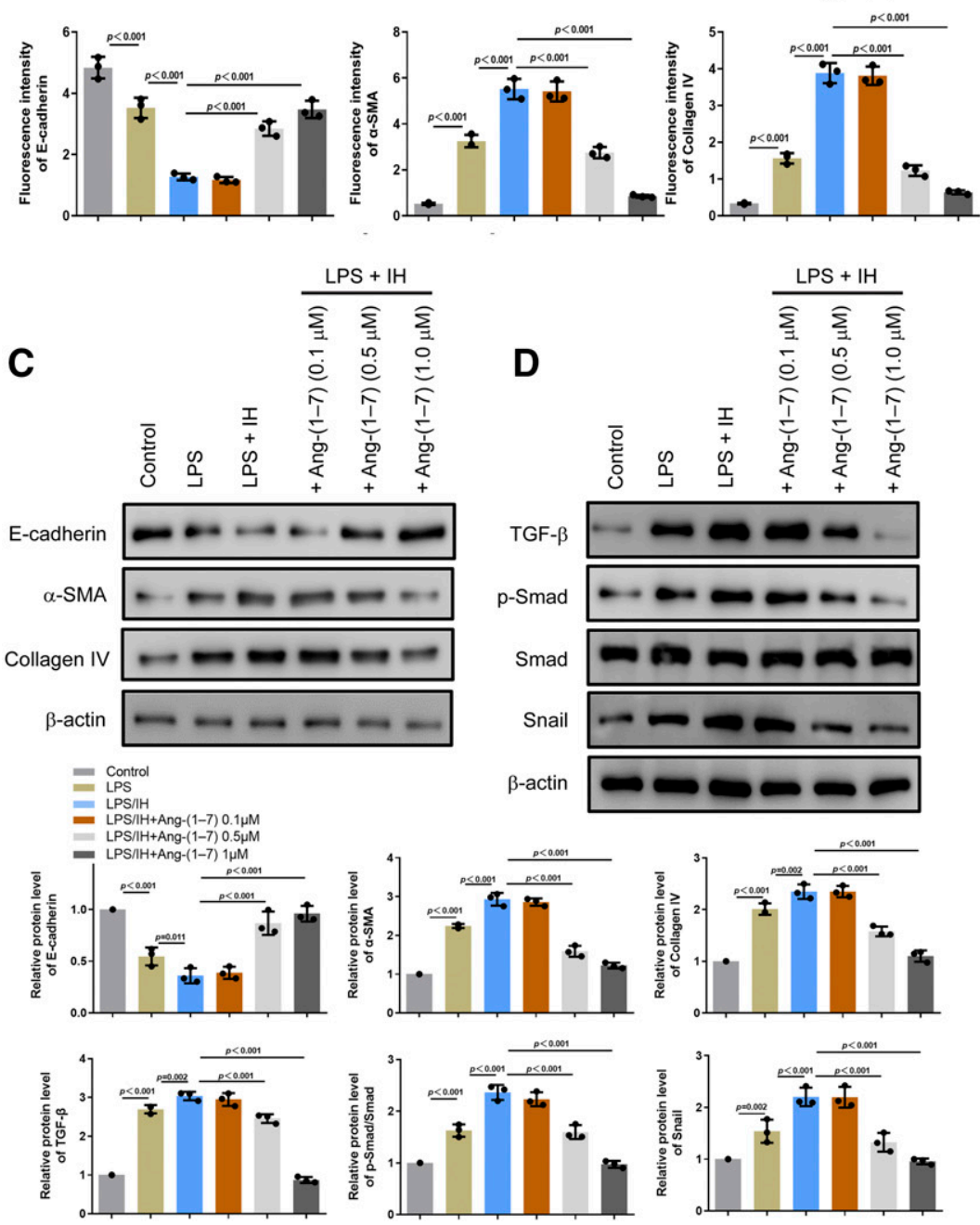

Fig. 3. Ang-(1-7) suppressed IH-induced TGF- $\beta$-mediated EMT in LPS-treated human bronchial epithelial cells. (A) IH decreased the level of E-cadherin and increased levels of $\alpha$-SMA and collagen IV in LPS $(10 \mu \mathrm{g} / \mathrm{ml})$-treated human bronchial epithelial BEAS-2B cells, which was reversed by administration of Ang-(1-7) (100 nM) in a dose-dependent manner. Representative images of immunofluorescence staining of BEAS-2B cells with antibodies against E-cadherin, a-SMA, and collagen IV were shown. Scale bar, $20 \mu \mathrm{m}$. (B) The average fluorescence intensity of E-cadherin, $\alpha$-SMA, and collagen IV was shown. Data are expressed as means \pm S.D. $(n=6)$. $* P:<0.05 ; * * P<0.01$; *** $P<0.001$ by one-way ANOVA followed by Tukey's multiple comparison test. (C) Representative Western blots with antibodies against E-cadherin, $\alpha$-SMA, and collagen IV in the indicated groups. $\beta$-Actin was used as the internal control. (D) IH increased the levels of TGF- $\beta$, p-Smad, and Snail in LPS (10 $\mu \mathrm{g} / \mathrm{ml})$-treated BEAS-2B cells, which was reversed by administration of Ang-(1-7) $(100 \mathrm{nM})$ in a dose-dependent manner. Representative Western blots with antibodies against TGF- $\beta$, p-Smad, Smad, and Snail were shown. $\beta$-Actin was used as the internal control.
ACE2/Ang-(1-7)/Mas axis protects against lung fibroblast migration and lung fibrosis (Meng et al., 2015). Exogenous Ang-(1-7), which counterbalances angiotensin II actions, directly inhibits EMT induced by TGF- $\beta$ in alveolar epithelial cells (Shao et al., 2019). Furthermore, studies have shown that chronic IH increased expression of angiotensin II and stimulates RAS (Fung, 2014). Exogenous Ang-(1-7) exerts renoprotective effects on chronic IH-induced renal injury by inhibiting fibrosis (Lu et al., 2017) and relieves chronic IHinduced lung injury (Lu et al., 2016). We found that Ang-(1-7) 
A
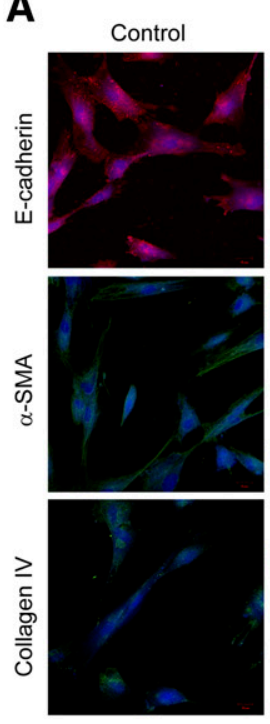

B
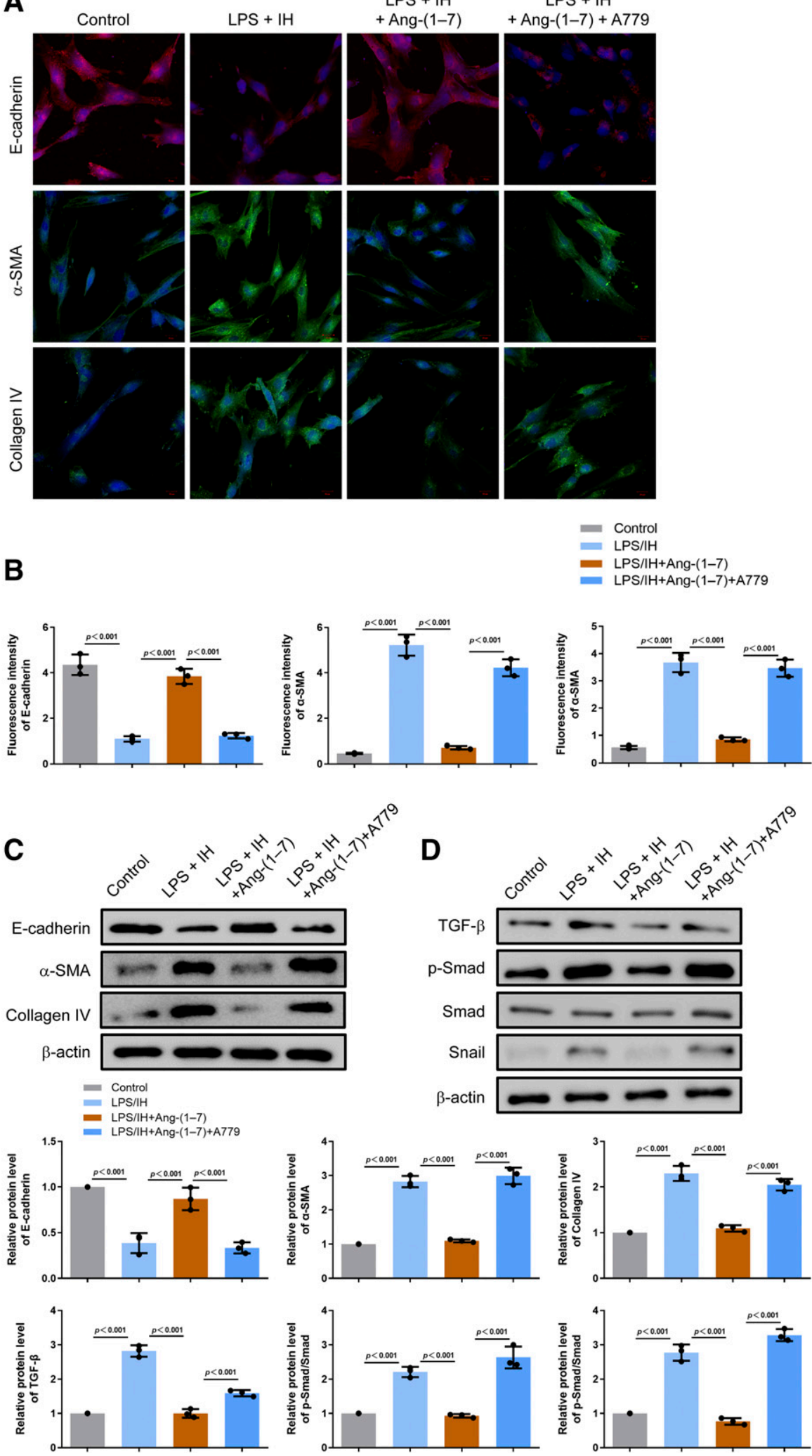

Fig. 4. A779 blocked the suppressive effects of Ang-(1-7) on TGF- $\beta$-mediated EMT upon IH exposure in LPS-treated human bronchial epithelial cells. (A) Representative images of immunofluorescence staining of BEAS-2B cells with antibodies against E-cadherin, $\alpha$-SMA, and collagen IV were shown. Scale bar, $20 \mu \mathrm{m}$. (B) The average fluorescence intensity of E-cadherin, $\alpha$-SMA, and collagen IV was shown. Data are expressed as means \pm S.D. $(n=6)$. $* P:<0.05$; $* * P<0.01$; *** $P<0.001$ by one-way ANOVA followed by Tukey's multiple comparison test. (C) Representative Western blots with antibodies against E-cadherin, $\alpha$-SMA, and collagen IV in the indicated groups. $\beta$-Actin was used as the internal control. (D) Representative Western blots with antibodies against TGF- $\beta$, p-Smad, Smad, and Snail were shown. $\beta$-Actin was used as the internal control. abolished the effects of chronic IH on TGF- $\beta$-mediated EMT, which could be blocked by the addition of specific Mas receptor antagonist A779, suggesting that the ACE2/Ang-(1-7)/Mas axis is involved in chronic IH-induced EMT in asthma.
In conclusion, chronic IH aggravates TGF- $\beta$-mediated EMT, which is attenuated by Ang-(1-7). These findings suggest that RAS activity is involved in the mechanisms of OSA exacerbating airway dysfunction in asthma. 


\section{Authorship Contributions}

Participated in research design: Zhou, Lin, Q.Y.Li

Conducted experiments: Zhou, Lin.

Performed data analysis: Zhou, Lin, N. Li, Sun, Ding, Yan, Zhang.

Wrote or contributed to the writing the manuscript: Zhou, Lin, Q.Y. Li.

\section{References}

Broytman O, Braun RK, Morgan BJ, Pegelow DF, Hsu PN, Mei LS, Koya AK Eldridge M, and Teodorescu M (2015) Effects of chronic intermittent hypoxia on allergen-induced airway inflammation in rats. Am J Respir Cell Mol Biol $\mathbf{5 2}$ 162-170.

Du W, Chen T, Ni Y, Hou X, Yu Y, Zhou Q, Wu F, Tang W, and Shi G (2017) Role of PIM2 in allergic asthma. Mol Med Rep 16:7504-7512.

Ferreira AJ and Santos RA (2005) Cardiovascular actions of angiotensin-(1-7). Braz J Med Biol Res 38:499-507.

Fung ML (2014) The role of local renin-angiotensin system in arterial chemoreceptors in sleep-breathing disorders. Front Physiol 5:336.

GINA (2020) From the global strategy for asthma management and prevention, Global initiative for asthma (GINA). https://ginasthma.org/reports.

Hackett TL (2012) Epithelial-mesenchymal transition in the pathophysiology of airway remodelling in asthma. Curr Opin Allergy Clin Immunol 12:53-59.

Hekking PP, Wener RR, Amelink M, Zwinderman AH, Bouvy ML, and Bel EH (2015) The prevalence of severe refractory asthma. J Allergy Clin Immunol 135:896-902.

Julien JY, Martin JG, Ernst P, Olivenstein R, Hamid Q, Lemière C, Pepe C, Naor N, Olha A, and Kimoff RJ (2009) Prevalence of obstructive sleep apnea-hypopnea in severe versus moderate asthma. J Allergy Clin Immunol 124:371-376.

Liu T, Liu Y, Miller M, Cao L, Zhao J, Wu J, Wang J, Liu L, Li S, Zou M, et al. (2017) Autophagy plays a role in FSTL1-induced epithelial mesenchymal transition and airway remodeling in asthma. Am J Physiol Lung Cell Mol Physiol 313:L27-L40.

Lu W, Kang J, Hu K, Tang S, Zhou X, Yu S, Li Y, and Xu L (2016) Angiotensin-(1-7) inhibits inflammation and oxidative stress to relieve lung injury induced by chronic intermittent hypoxia in rats. Braz J Med Biol Res 49:e5431.

Lu W, Kang J, Hu K, Tang S, Zhou X, Yu S, and Xu L (2017) Angiotensin-(1-7) relieved renal injury induced by chronic intermittent hypoxia in rats by reducing inflammation, oxidative stress and fibrosis. Braz J Med Biol Res 50:e5594.

Meng Y, Li T, Zhou G-S, Chen Y, Yu CH, Pang MX, Li W, Li Y, Zhang WY, and Li X (2015) The angiotensin-converting enzyme 2/angiotensin (1-7)/Mas axis protects against lung fibroblast migration and lung fibrosis by inhibiting the NOX4-derived ROS-mediated RhoA/Rho kinase pathway. Antioxid Redox Signal 22:241-258.

Millar EA, Angus RM, Hulks G, Morton JJ, Connell JM, and Thomson NC (1994) Activity of the renin-angiotensin system in acute severe asthma and the effect of angiotensin II on lung function. Thorax 49:492-495.

Qu ZH, Yang ZC, Chen L, Lv ZD, Yi MJ, and Ran N (2012) Inhibition airway remodeling and transforming growth factor- $\beta 1 /$ Smad signaling pathway by astragalus extract in asthmatic mice. Int $J$ Mol Med 29:564-568.

Sforza E and Roche F (2016) Chronic intermittent hypoxia and obstructive sleep apnea: an experimental and clinical approach. Hypoxia (Auckl) 4:99-108.

Shao M, Wen Z-B, Yang H-H, Zhang CY, Xiong JB, Guan XX, Zhong WJ, Jiang HL, Sun CC, Luo XQ, et al. (2019) Exogenous angiotensin (1-7) directly inhibits epithelial-mesenchymal transformation induced by transforming growth factor- $\beta 1$ in alveolar epithelial cells. Biomed Pharmacother 117:109193.

ten Brinke A, Sterk PJ, Masclee AA, Spinhoven P, Schmidt JT, Zwinderman AH, Rabe KF, and Bel EH (2005) Risk factors of frequent exacerbations in difficult-totreat asthma. Eur Respir $J$ 26:812-818.

Tian X, Tian X, Huo R, Chang Q, Zheng G, Du Y, Chen Y, and Niu B (2017) Bacillus Calmette-Guerin alleviates airway inflammation and remodeling by preventing TGF- $\beta_{1}$ induced epithelial-mesenchymal transition. Hum Vaccin Immunother 13: $1758-1764$

Trevor JL and Deshane JS (2014) Refractory asthma: mechanisms, targets, and therapy. Allergy 69:817-827.

Wang Z, Zhang H, Sun X, and Ren L (2016) The protective role of vitamin D3 in a murine model of asthma via the suppression of TGF-B/Smad signaling and activation of the Nrf2/HO-1 pathway. Mol Med Rep 14:2389-2396.

Yang ZC, Yi MJ, Ran N, Wang C, Fu P, Feng XY, Xu L, and Qu ZH (2013) Transforming growth factor- $\beta 1$ induces bronchial epithelial cells to mesenchymal transition by activating the Snail pathway and promotes airway remodeling in asthma. Mol Med Rep 8:1663-1668.

Yigla M, Tov N, Solomonov A, Rubin AH, and Harlev D (2003) Difficult-to-control asthma and obstructive sleep apnea. J Asthma 40:865-871.

Zhou JP, Tang W, Feng Y, Li N, Gu CJ, Li QY, and Wan HY (2016) Angiotensin-(1-7) decreases the expression of collagen I via TGF- $31 / \mathrm{Smad} 2 / 3$ and subsequently inhibits fibroblast-myofibroblast transition. Clin Sci (Lond) 130:1983-1991.

Address correspondence to: Qing Yun Li, Department of Respiratory and Critical Care Medicine, Ruijin Hospital, Shanghai Jiao Tong University School of Medicine, Shanghai 200025, China. E-mail: liqingyun68@hotmail.com 